\section{Reliable Dosing system}

W

alther Systemtechnik has developed a new dosing system for precision dosing of substances with widely varying viscosities, such as solvents, silicones and epoxy resin adhesives.

According to the manufacturer, this system produces better results than comparable conventional systems. Its special features include a fast solenoid, pressure and vacuum sensors, a highly accurate digital time and pressure display and a precision pressure regulator.

In addition, the machine has a teach function that allows the operator to store the dosing volume and time so that the results can be easily reproduced.

It can be used to control pneumatic and electro-pneumatic valves supplied by Walther, as well as many other com- mon types of valves available on the market.

An interface is available to allow the machine to be integrated into semi-automatic and fully automatic systems

For more information, please contact: Walther Systemtechnik GmbH, D-76726 Germersheim, Germany, www.walther-2000.de

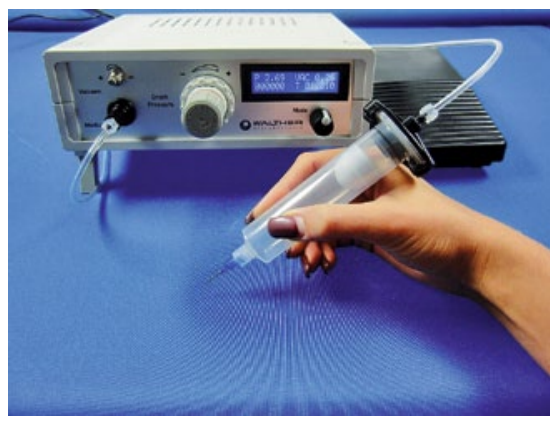

\section{Continuous Manufacturing of Highly Viscous Products}

$\Lambda$ s manufacturers of highly viscous prodAucts such as sealants and insulating compounds are aware, the critical stage in the production process involves mixing fine particulate solids with a viscous fluid. Costly extruding machines with a high energy consumption are generally used for this pur-

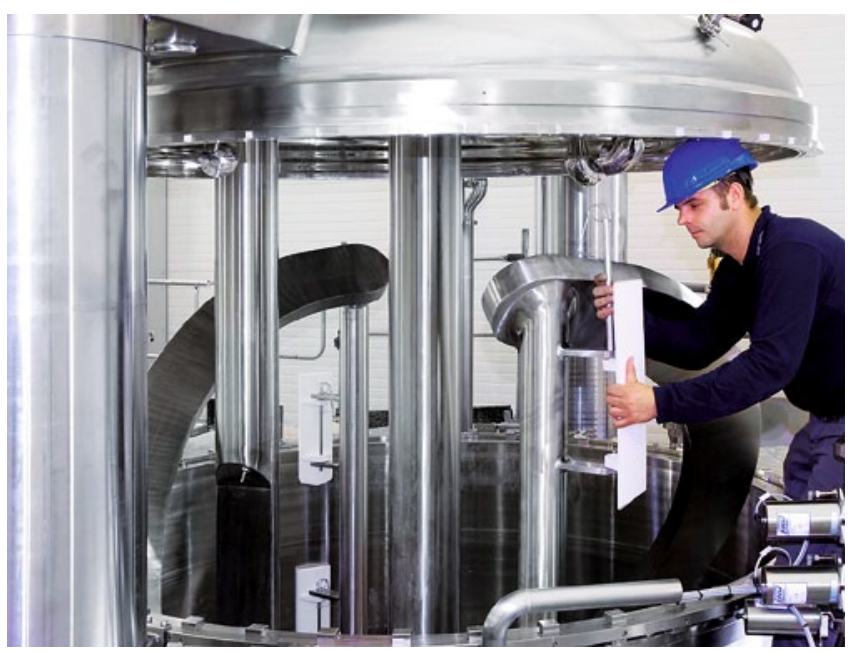

pose. Ekato RMT has developed a new machine concept which significantly reduces the energy used in this process. The mixing tools are designed to divide the container, through which a continuous flow of materials passes into two parts: an intensive backmixing area and an area of high shear intensity. The solids are wetted in the mixing area and then fully dispersed in the shear area with plug flow behaviour, which ensures consistently high product quality.

For more information, please contact: Ekato Holding $\mathrm{GmbH}$, info@ ekato.com, www. ekato.com

\section{VAE Dispersion for Difficult-To-Bond Surfaces}

$\bigvee_{\text {persion Vinnapas EP } 8010 \text { spe- }}^{\text {acker has developed the dis- }}$ cifically for bonding various surfaces in the paper and packaging industry. Based on vinyl acetate-ethylene (VAE) copolymers, it reliably and permanently bonds challenging paper and cardboard surfaces, such as coated or painted paper, and polymeric film. According to the manufacturers, apart from its excellent adhesive strength, the new product is characterised by

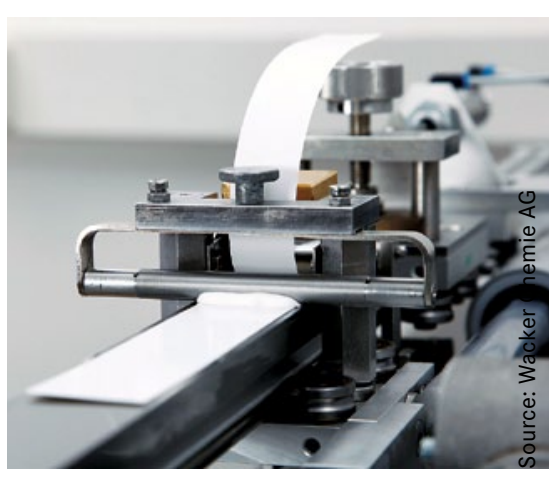

Testing the setting speed

outstanding heat resistance, a very high setting speed and ease of machine processing. Moreover, it is manufactured without the use of alkylphenol ethoxylates (APEO), and its VAE basis makes supplementary plasticizers unnecessary. This dispersion is therefore ideal for formulating highperformance adhesives for demanding paper and packaging applications in which difficult-to-bond substrates have to be permanently joined.

The new dispersion will be unveiled at the World Adhesive \& Sealant Conference (WAC) 2012, which will take place from 18 to 21 September in Paris, France.

For further information, please contact: Wacker Chemie AG, D-81737 Munich, www.wacker.com 